\title{
Design and Implementation of Mobile Blended Learning Model Based on WeChat Public Platform
}

\author{
Yanyan Han, Liyue Tian and Weijun Cheng \\ College of Information Engineering, Minzu University of China, Beijing, China
}

\begin{abstract}
Merging together the ideas of mobile learning, blended learning and flipped classroom, a Mobile Blended Learning Model (MBLM) is constructed. Based on WeChat Public Platform (WPP), MBLM can optimize the instructional process and improve the learning efficiency. A Mobile Blended Learning System (MBLS) is implemented by using MBLM, and it is constructed by both WPP and auxiliary learning system which based on Java Web. This system has reasonable designed function, easy operation, and beautiful interface, so it can effectively promote the popularization of MBLM.
\end{abstract}

\section{Introduction}

In 2012, the idea of "Internet + " was proposed, and brought revolutionary impact and challenge to the traditional educational idea [1]. Blended learning aims to find the correct balance between face-to-face and online teaching methodologies. This also integrates well with the idea of a "flipped classroom" [2]. The prevalence of mobile Internet has optimized the traditional online learning model, and learning will move to a larger extent out of the classroom [3]. Blended learning transformed from one with passive students who were recipients of knowledge, as is typical of traditional teaching models, to one with active students who constructed their own knowledge [4]. Since 2011, study of WeChat has presented an explosive growth. But now, few studies can apply a variety of functions of WeChat and WeChat Public Platform (WPP) to the whole instructional process.

Therefore, Mobile Blended Learning Model (MBLM) based on WPP is proposed. It integrates the idea of blended learning, mobile learning, and flipped classroom. Mobile Blended Learning System (MBLS) combines WPP and Java program. It can effectively promote the implementation of MBLM based on WPP and make it more convenient to use by learners.

\section{MBLM based on WPP}

The affordances of blended learning are now well understood, and its flexibility, ease of access, and the integration of sophisticated multimedia and technologies are high among the list of appeals [5]. Rong-huai Huang [6] et al. proposed "Design framework of Blended Learning Curriculum" in 2009. According to this framework, design of blended learning model should be divided into three stages: pre-phase analysis, design of activities and resources, and instructional evaluation design. In the third stage, the design of activities and resources can best embody the characteristics of blended learning. This design is guided by the basic theory of pedagogy, supported by the function of WPP, and took time as horizontal coordinate. We combine WPP and traditional classroom, optimize and design the MBLM based on WPP in two 
areas: learners and teachers. As showed in Figure 1, we will emphatically introduce the system design of online learning based on WPP as bellow.

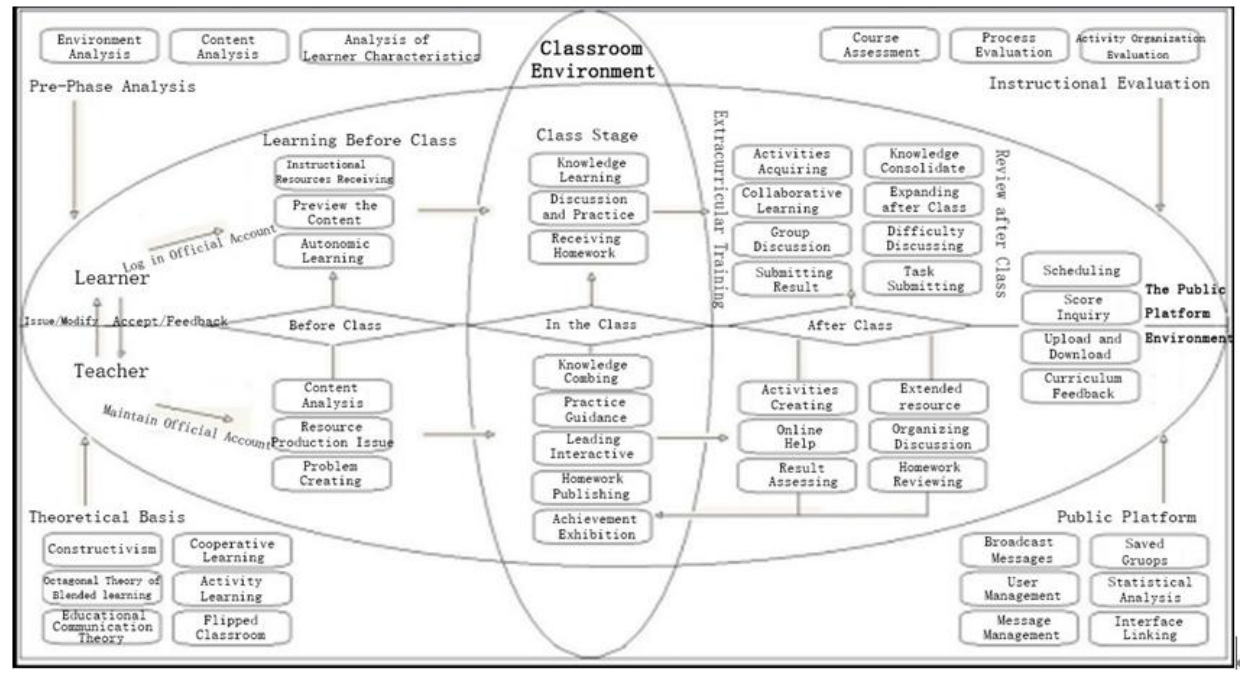

Fig. 1. MBLM Based on WPP Based on WeChat Official Platform.

There are a great many functions of WPP, such as interface, customize menus, management of Broadcast Groups, user management, information management, statistical analysis and so on. MBLM based on WPP uses the interface function of WPP. These functions assist the development of the whole blended learning.

The first stage is pre-class analysis. Before the curriculum, it is necessary to in-depth analyze the learning environment, learning content, and the characteristics of the learners, to determine whether the course is suitable for the development of MBLM.

Then, the next stage is Design of Activities and Resources. blended learning provides anytime anywhere access to rich learning materials and course content over the Internet[8]. Design of activities and resources can be divided into pre-class learning, instructional stage, after-class review, and extracurricular activities, training, implementation. Before class, the teacher releases new learning content framework, difficulty, provides relevant learning materials and instructional video and other multimedia courseware. In class, the teacher systematically summarizes the previewing knowledge and publishes the relevant information needed for class. After class, the teacher releases knowledge summaries, extracurricular activities, experiment and extracurricular learning materials, and collects the assignments and test papers to review corrections in the WPP. At the same time, the teacher will organize the discussion in the Saved Groups once more.

The instructional evaluation design is the last stage of the whole instructional process, mainly including curriculum evaluation, process evaluation, and activity organization evaluation, and they are respectively measured through the online testing, the situation of the discussion in the classroom, and activity experimental review.

\section{Design of MBLS}

The system is mainly based on WPP, and supplemented by the Java Web auxiliary system, combining with the Instant Messaging, Saved Groups, Moments, Official Account Security and other WeChat functions. The system aims realizes instant messaging, online testing, upload and download of related document, query results, course feedback, student management and statistical and other functions, then construct a multiple learning system based on WeChat.

Implementation of related functions fully relies on WPP and WeChat's basic functions, and the interface style and operational methods are consistent with WeChat's office. Auxiliary system requires rational design of the system, clear thinking, complete functions, and easier operation. Because the system is mainly used in mobile phone terminal, the 
interface design should be simple, and options should be clear and easy to operate. In addition, the use of support tools can improve the whole system. Official Account Security can meet the instant management function. Cloud notes and SkyDrive can accomplish online management and sharing of notes and documents. Analysis function of Public Platform can be used to analyze the data. WeChat Saved Groups' instant messaging feature can be used to discuss questions, as well as WeChat Moments can be shared promotion. Based on the above, the entire MBLS is constructed as showed in Figure 2.

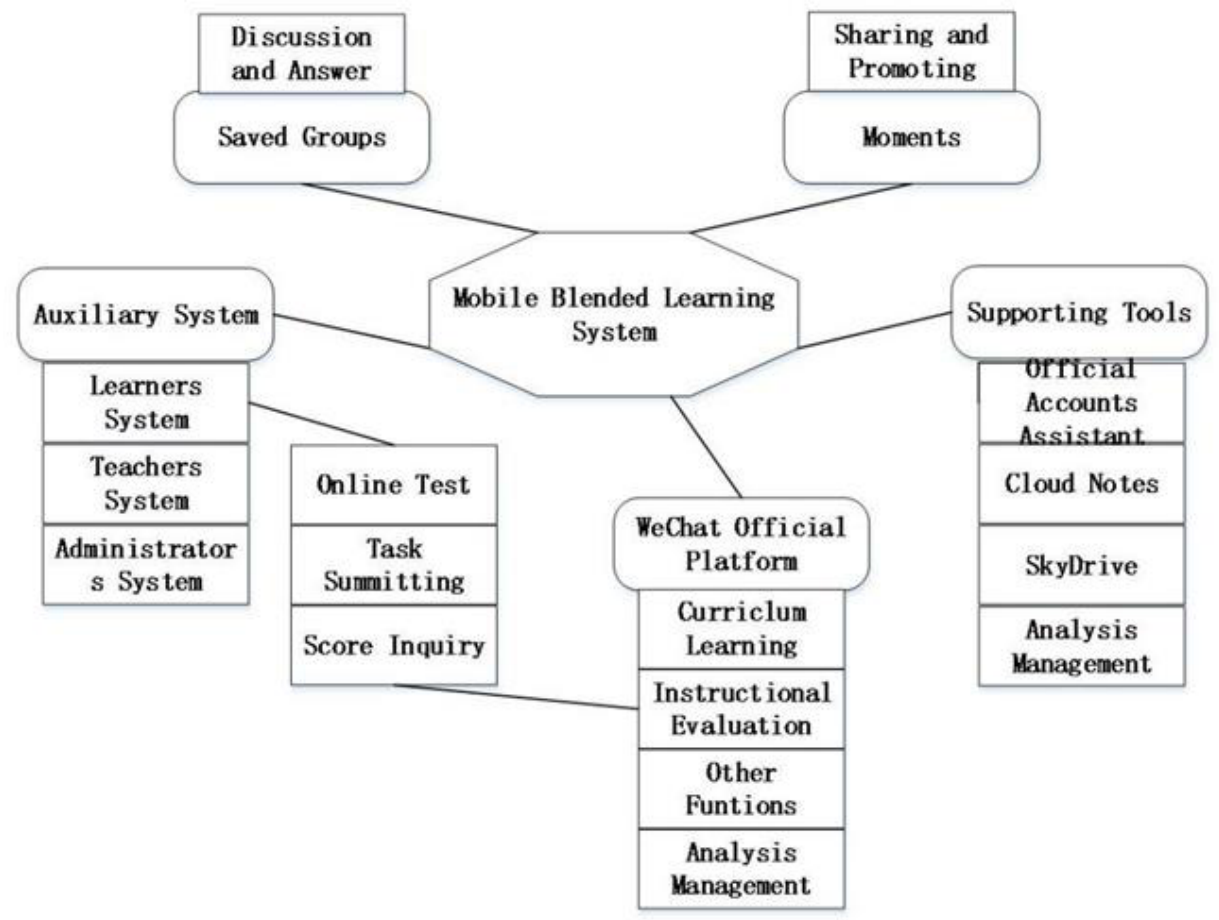

Fig. 2. Schematic diagram of MBLS.

\subsection{WPP system}

The system is divided into four modules: curriculum learning, instructional evaluation, analysis management, and other functions. In view of this, system functions decomposition diagrams can be designed as showed in Figure 3.

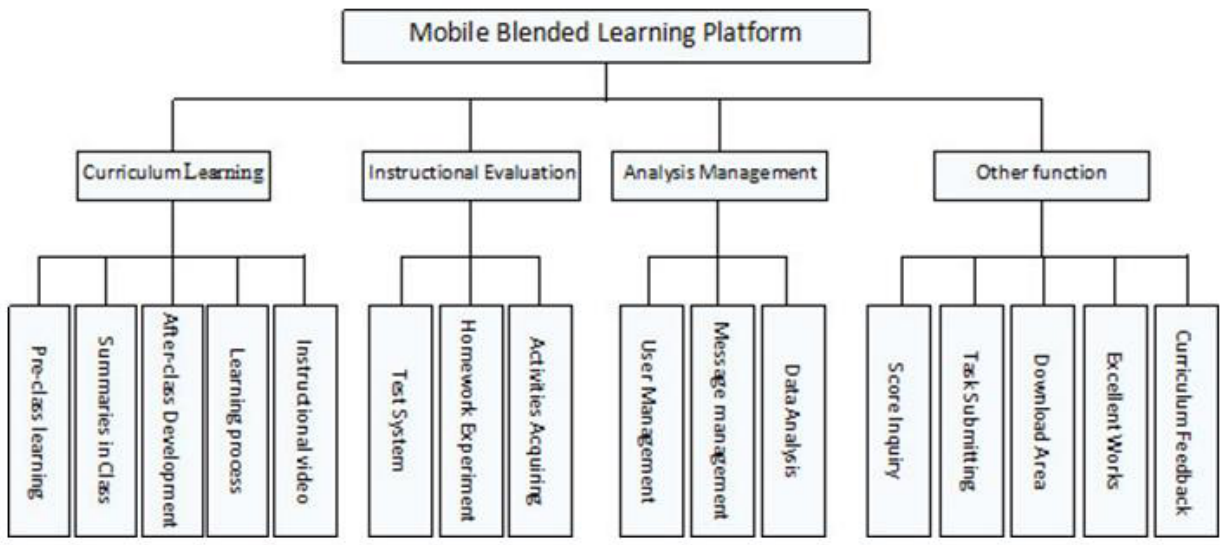

Fig. 3. Functional decomposition diagrams of WPP system. 
Curriculum learning mainly consists of five modules: Pre-class Learning, summaries in class, After-class Development, Learning Progress, and Instructional Video. Through clicking on the submenus of WeChat platform, the teacher can present the learning resource needed to the learner, in image-text or video form.

By extending the classroom space to the online space for interactions, teacher noticed the importance of prompt feedback [7]. Instructional evaluation is accomplished through test, homework, experiment, activity and etc. Information is released by clicking on the submenu in image-text form. Because the function of test is unable to realize based on WPP, this design implements this function by clicking on the submenu to jump to an auxiliary system.

Through the functions of user management, message management and data analysis, teachers (or administrators) can realize the functions of message handling, instructional data analysis and student management.

The system sets up other auxiliary functions, to better serve the entire learning process. The functions of results submitting and score query are implemented through auxiliary system, and the functions of download and curriculum feedback can be achieved through clicking on the submenu.

\subsection{Auxiliary system based on Java Web}

This system implements some functions, such as online testing, upload and download of related document, performance and other related information query and other functions to enrich the whole MBLS through design of auxiliary system based on Java Web. This system users are divided into three categories: administrator, teacher and learner. The detailed functions of these three module is shown in Figure 4.

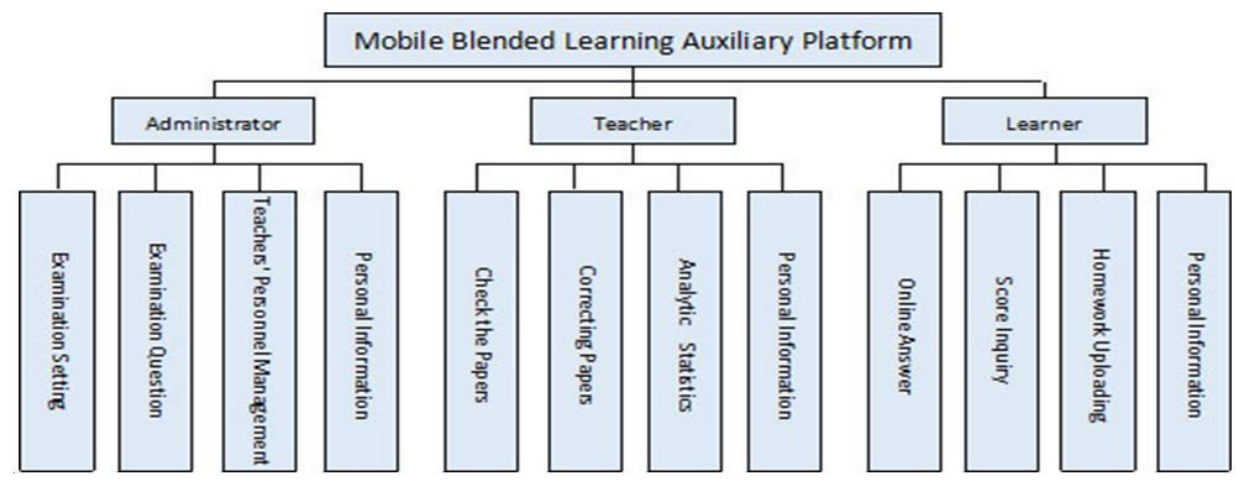

Fig. 4. Functional decomposition diagrams of Auxiliary System.

\section{Implementation of MBLS}

\subsection{Implementation of WPP}

You could register an Official Account and $\log$ in the official website of the WPP. Then you could see the homepage with managing and editing function, as shown in Figure 5. 


\begin{tabular}{|c|c|c|}
\hline \multicolumn{3}{|l|}{ II: Fuction } \\
\hline Fsend & $\Omega 6$ & 0,214 \\
\hline AutoReply & NewMessage & TotalNumber \\
\hline \multicolumn{3}{|l|}{ Customize Menus } \\
\hline Poll Manage & System Announcement & \\
\hline + Add plug-in 페 & WeChat public platform small program QR code is online. & $2016-12-30$ \\
\hline A. Management & WeChat public platform small program QR code add new function. & $2016-12-21$ \\
\hline Message & User management optimization, and support tag function. & $2016-12-19$ \\
\hline Customer & 2017 WeChat open class Pro version is grabing votes. & $2016-12-12$ \\
\hline Material & WeChat public platform launches new customer service & 2016-12-06 \\
\hline Al Extension & WeChat publishes web design style library & $2016-11-16$ \\
\hline Advertiser & WeChat public platform small program opens beta program. & 2016-11-03 \\
\hline FlowBenefit & Public platform released WeChat Web Developer Tools. & 2016-09-12 \\
\hline (c) Statistics & WeChat public platform "sweep" function opens beta program. & 2016-09-09 \\
\hline
\end{tabular}

Fig. 5. Management editing interface of Official Account.

\subsection{Implementation of auxiliary system}

This system is a Java Web project based on B/C model, with SQLServer2005. The server uses Tomcat v8.0 to accomplish local test. And as the need for linking with WeChat Official Account, it is necessary to purchase the domain name, and lend the server and publish it on the line. For the system is not commercially available, and taking into account the problems related to cost, this test applies for free domain using peanut shell software, and uses the port mapping method to temporarily visit this system in the public net.

\section{Conclusion}

MBLM based on WPP optimizes the instructional process and improves the learning efficiency. MBLS uses WPP and its convenient third-party interface to implement MBLM. With enormous user groups, excellent instant messaging, rich present form of content, cross-platform, free and easy constructing and other advantages, WPP provides teachers with solutions which are universal, simple operation, strong extensibility. At the same time, with the open interface teachers can develop and extend functions to improve the feasibility of MBLM. But now, the design and implementation of MBLM based on WPP has not yet added the factors of subject. In the future research, we will further combine with the characteristics of different subjects, to implement MBLM based on WPP in different situations.

\section{References}

1. Y. Zhang. "Internet+ Education" idea and pattern analysis. China's higher EDU. RSRCH. 02,70-73,(2016)(in Chinese)

2. C.E. Morton, S.N. Saleh, S.F. Smith et.al. Blended learning: how can we optimise undergraduate student engagement? Bmc Medical EDU. 16(1),(2016)

3. Sheng-quan Yu. From the knowledge transfer to cognitive construction, and situational awareness---Development and outlook of the three generations of mobile learning. China EDU TECH. 6,(2007) (in Chinese)

4. E.M.T. Maza, M.T.G. Lozano, A.C.C. Alarcón et al. Blended learning supported by digital technology and competency-based medical education: a case study of the social medicine course at the Universidad de los Andes, Colombia. International JRNL. EDU. TECH. Higher EDU. 13(1),2016 
5. NMC Horizon Report (2016 Higher Education Edition). (2016)

6. R.H. Huang, D. Ma, L.Q. Zheng. Based on blended learning theory of curriculum design. E-EDU. RSRCH. (1):11-16,(2009) (in Chinese)

7. H. Çakir, B.A. Bichelmeyer. Effects of teacher professional characteristics on student achievement: an investigation in blended learning environment with standards-based curriculum. Interactive Learning ENVR. 24(1):1-13,(2013)

8. M. Lai, K.M. Lam, C.P. Lim. Design principles for the blend in blended learning: a collective case study. Teaching in Higher EDU. 1-14(2016) 